\title{
An Approach for Image Enhancement based on Improved Joint Bilateral Retinex
}

\author{
Thillai Sivakavi Saravanan ${ }^{1}$ \\ ${ }^{1}$ Department of Information Technology, \\ Panimalar Institute of Technology, \\ No.391, Bangalore Trunk Road, Varadharajapuram, \\ Poonamallee, Chennai - 600 123, Tamil Nadu, India
}

Abstract: Image enhancement is a crucial technique to accentuate images with poor illumination in order to achieve better image interpretation. Retinex Theory is a classic Image enhancement technique for improving poor illumination in the digital image. In this work an experiment is carried for the improvement of poor illumination in an image to aid better enhancement based on retinex theory with improved joint bilateral filter which enhances the poor illumination of digital images without compromising the edges. From the results it is observed that the combination of retinex theory with joint bilateral filters aids better image enhancement.

Keywords: Retinex theory; digital image; bilateral; image enhancement; illumination.

\section{INTRODUCTION}

Image Enhancement plays a vital role in many image processing applications. The idea behind image enhancement is to improve the given image to be a "better" image by applying some technique for better human interpretation or to send as input for further analysis [1]. Some of the image enhancements techniques comprise of Wavelet transform, Gamma Correction, Spatial Filtering techniques and Histogram Equalization [2].Retinex theory is the classic image enhancement technique which is proposed by Land [3] that aids better image enhancement by improving the poor illumination in the image. To handle halo effect of retinex theory and to achieve colour restoration joint bilateral filter can be used. The Joint Bilateral Filter which is proposed by Eisemann and Durand [4] an altered edition of bilateral filter which splits the impression of edges and protects it to get smoothened.

\section{LITERATURE}

Li Yang et al [5] proposed a trilateral filter based retinex for the image contrast enhancement and achieved a good solution. Ling Tang et al [6] proposed an improved retinex theory for the image enhancement and from the results it is concluded that the proposed method achieves improved image enhancement. Nirmal Jith et al [7] proposed a denoising algorithm based on joint bilateral filter and achieved better denoising for high noise image. Wan de et al [8] proposed enhancement technique for the image enhancement for local mode filtering. Xueyang $\mathrm{Fu}$ et al [9] proposed a method to eliminate uneven illumination and achieved better results when compared with the existing methods. Shi Tang et al [2] proposed a colour image enhancement based on retinex theory with guided filter and achieved improved result in handling

\author{
Shenbaga Priya $\mathrm{V}^{2}$ \\ ${ }^{2}$ Computer Applications
}

School of Computer Information and Mathematical Sciences B.S. Abdur Rahman Crescent Institute of Science \& Technology GST Road, Vandalur, Chennai 600 048, Tamil Nadu, India.

halo effect and image loss when comparing the existing results.

\section{BASIC RETINEX METHODS}

The role of retinex theory is to decouple an image into two component, they are illumination and reflectance. The retinex theory reduces the impact of illumination of the reflectance. The main ideology behind the retinex is reconstruction of image which mimics human visual system and an image can be defined as follows:

$$
I_{n}(x, y)=L_{n}(x, y) \cdot R_{n}(x, y)
$$

Where $I_{n}(x, y)$ is the given image, $L_{n}(x, y)$ Illumination of the given image and $R_{n}(x, y)$ is the reflectance of the object. In order to enhance the image the illumination compound has to be suppressed and the reflectance compound has to be maintained that can be defined as

$$
R_{n}(x, y)=\frac{I_{n}(x, y)}{L_{n}(x, y)}
$$

Logarithmic function are applied on both sides for the convenience to the equation (2)

$$
\log R_{n}(x, y)=\log I_{n}(x, y)-\log L_{n}(x, y)
$$

$L_{n}(x, y)$ can be estimated by wrapping $I_{n}(x, y)$ with the gaussian function $G_{\sigma}(x, y)$.

$$
I_{n}(x, y)=L_{n}(x, y) * G_{\sigma}(x, y)
$$

Where $\sigma$ is the guassian function standard deviation,"*" denotes the convolution operation. $\mathrm{G}_{\sigma}(\mathrm{x}, \mathrm{y})$ can be defined as

$$
G_{\sigma}(x, y)=\frac{1}{\sqrt{2 \pi} \sigma} \cdot \exp \left(\frac{x^{2}+y^{2}}{2 \sigma^{2}}\right)
$$

While substituting equation 4 and equation 5 in equation 3 resulting in Single Scale Retinex (SSR).

$$
\begin{gathered}
\log R_{n}(x, y)=\log I_{n}(x, y)-\log \left[I_{n}(x, y) *\right. \\
\left.G_{\sigma}(x, y)\right]
\end{gathered}
$$

The reflection compound $\mathrm{R}_{\mathrm{n}}(\mathrm{x}, \mathrm{y})$ is stated as 


$$
R_{n}(x, y)=255 \cdot \frac{\log R_{n}(x, y)-\min \left(\log R_{n}(x, y)\right)}{\max \left(\log R_{n}(x, y)-\min \left(\log R_{n}(x, y)\right)\right)}
$$

Where $\min \left(\log R_{n}(x, y)\right)$ is minimum of $\log R_{n}(x, y)$ and where $\max \left(\log R_{n}(x, y)\right)$ is maximum of $\log R_{n}(x, y)$.

The multiscale retinex is defined as:

$$
R_{M S R}(x, y)=\sum_{n=1}^{N} \omega_{n} R_{n}(x, y)
$$

$$
\begin{aligned}
R_{M S R}(x, y)= & \sum_{\substack{n=1\\
}}^{N} \omega_{n}\left\{\log I_{n}(x, y)-\log \left[I_{n}(x, y)\right.\right. \\
& \left.\left.* G_{\sigma}(x, y)\right]\right\}
\end{aligned}
$$

Where $\mathrm{N}$ is the number of SSR[2].

\section{PROPOSED IMPROVED JOINT BILATERAL RETINEX METHOD}

Different shades of retinex image enhancement techniques have evolved to handle unevenness in the images. Halos and smoothening of edges is still a challenging issue in retinex theory. Usage of Filtering technique can protect the images from halos and excessive smoothening. In this section retinex theory with joint bilateral filter has been experimented to achieve improved image enhancement based on retinex technique. Figure 1 depicts the process flow of the proposed method.

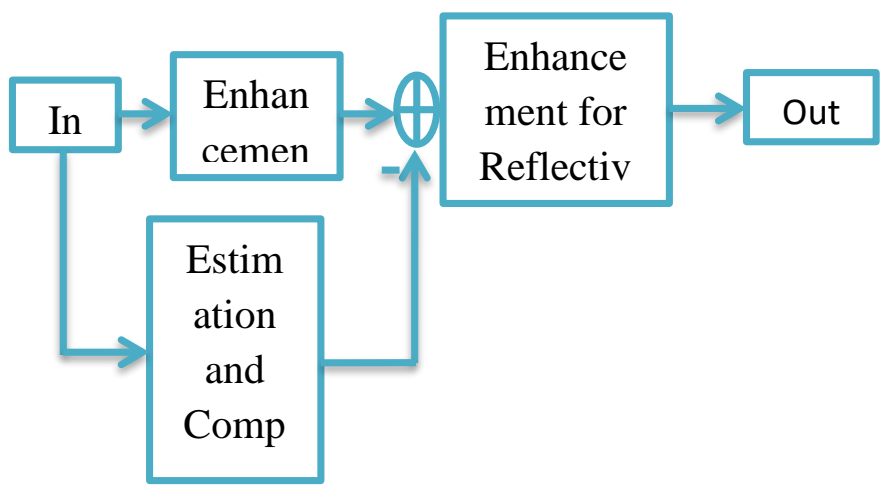

Figure 1 Joint Bilateral Retinex Process Flow.

First the given image is transformed to RGB to HSI(Hue,Saturation,Intensity) space. Gamma corrections are used to improve the channel I. It is defined with power expression.

$$
g(i)=i^{\gamma} \mathrm{c}
$$

Where $\mathrm{i}$ is raised to the power of gamma. $\mathrm{x}$ is a non-negative real input value and multiplied with a constant c. Gamma correction is used to enhance the channel $\mathrm{i}$ as in equation 10.
Where $\mathrm{G}(\mathrm{x}, \mathrm{y})$ gamma corrected image.G' $(\mathrm{x}, \mathrm{y})$ is the actual image. Estimation of illumination image is carried with joint bilateral filter to supress the colour degradation [10][11][12].

$$
\begin{gathered}
f[x]_{p=\frac{1}{W_{p}} \sum_{q \in \Omega} G_{\sigma_{s}}(\| p-} \\
q \|) \cdot G_{\sigma_{r}}\left(\left|Y_{p}-Y_{q}\right|\right) \cdot x_{q}
\end{gathered}
$$

Where $[\mathrm{x}] \mathrm{p}$ is the image value at $\mathrm{p} . \mathrm{Wp}$ is the normalization factor ensures pixels weight sum is 1.0.Generally bilateral filter ensures two weights viz.,the geometric weight $G_{\sigma_{s}}$ in the spatial domain. $G_{\sigma_{r}}$ Photometric weight in the range domain. $\sigma_{\mathrm{s}}$ and $\sigma_{\mathrm{r}}$ determines the filtering level. $\mathrm{F}[\mathrm{x}] \mathrm{p}$ is the target image $\mathrm{Y}$ is the given image. $\|\mathrm{p}-\mathrm{q}\|$ is the Euclidian distance between the pixel location $\mathrm{P}$ and $\mathrm{Q}$. In Joint bilateral filter a guidance image is used instead of filtered image. Compression for the illuminant image is defined as follows:

$$
f^{\prime}[x]_{=} g(f(x))
$$

Decomposition and enhancement of the reflected image is defined as:

$$
\begin{gathered}
R(x, y)=g^{\prime}(x, y)-\beta f^{\prime}[x] \\
R^{\prime}(x, y)=\bar{g}(x, y)+k *[g(x, y)- \\
\bar{g}(x, y)]
\end{gathered}
$$

The brightness of output and input is represented in $\mathrm{g}^{\prime}(\mathrm{x}, \mathrm{y}), \mathrm{g}(\mathrm{x}, \mathrm{y}) \cdot \overline{\mathrm{g}}(\mathrm{x}, \mathrm{y})$ represents neighbourhood mean of $3 \mathrm{x} 3$ region. $\mathrm{K}$ is the gain co-efficient between 0 and 1.Then adjustment of $\mathrm{S}$ channel is defined as

$$
\begin{gathered}
s^{\prime}(x, y)=s(x, y)+t\left(G^{\prime}(x, y)-\right. \\
G(x, y)) * \lambda(x, y)
\end{gathered}
$$

Where $s^{\prime}(x, y)$ corrected saturation and $s(x, y)$ represents the uncorrected saturation. The coefficient proportional between 0 and 1 is represented as $t$.

\section{RESULTS AND DISCUSSION}

The proposed method is implemented in MATLAB and validated with the existing techniques like Single Scale Retinex and Multi Scale Retinex. PSNR and MSE are used as validation parameter. Figure 2 shows the results of proposed method and other existing technique.

$$
G^{\prime}(x, y)=g(G(x, y))
$$




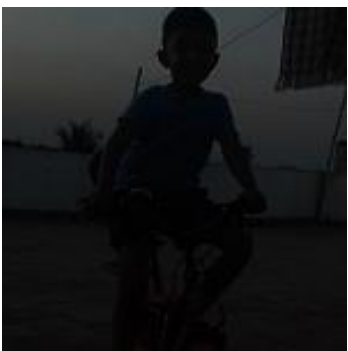

a)Actual Picture

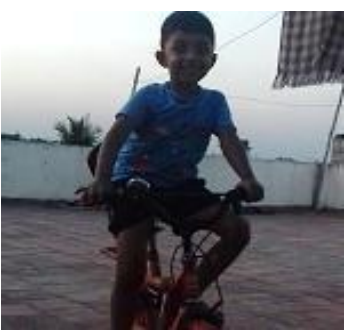

c)Multiscale Retinex

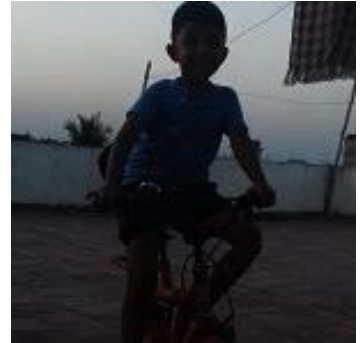

b)Single Scale Retinex

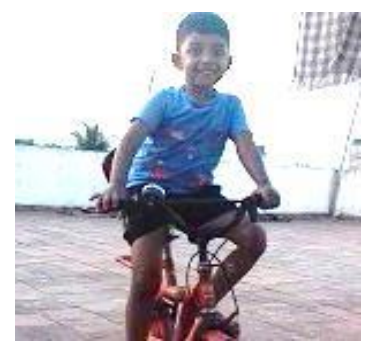

d)Proposed Method
Figure 2 Output of Proposed Method With the Existing Methods

\section{A. Peak Signal to Noise Ratio(PSNR):}

PSNR technique is a quality measure that is used in computing the compression ratio between actual and the proposed image.

$$
P S N R=10 \log _{10}\left[\frac{255^{2}}{M S E}\right]
$$

\section{B. Mean Square Error(MSE):}

Mean Square Error is the error metrics used to find the compression ratio. The lower the value of MSE the lower the error.

$M S E=\frac{1}{M \times N} \sum_{i=1}^{M} \sum_{i=1}^{N}\left(X^{\prime}(i, j)-X(i, j)\right)^{2}$

\begin{tabular}{|c|c|c|}
\hline Methods & $\begin{array}{c}\text { PSNR Value of the } \\
\text { Image }\end{array}$ & MSE value of the Image \\
\hline $\begin{array}{c}\text { Single Scale } \\
\text { Retinex }\end{array}$ & 13.295 & 0.445 \\
\hline $\begin{array}{c}\text { Multi Scale } \\
\text { Retinex }\end{array}$ & 13.987 & 0.223 \\
\hline Proposed Method & 14.334 & 0.222 \\
\hline
\end{tabular}

Table 1 Results of the proposed method and the existing method

\section{CONCLUSION}

The proposed image enhancement method effectively improves the poor contrast of the given image. In this paper a Joint Bilateral Retinex is experimented and evaluated based on the metrics PSNR and MSE. From the results it is concluded that the proposed method achieves better image enhancement when compared with the existing method.

\section{REFERRENCES}

[1] Bedi SS, Khandelwal R. Various image enhancement techniques-a critical review. International Journal of Advanced Research in Computer and Communication Engineering. 2013 Mar;2(3).

[2] Tang S, Dong M, Ma J, Zhou Z, Li C. Color image enhancement based on retinex theory with guided filter. In2017 29th Chinese Control And Decision Conference (CCDC) 2017 May 28 (pp. 5676-5680). IEEE.

[3] Land EH, McCann JJ. Lightness and retinex theory. Josa. 1971 Jan $1 ; 61(1): 1-1$.

[4] Paris S, Kornprobst P, Tumblin J, Durand F. Bilateral filtering: Theory and applications. Foundations and Trends in Computer Graphics and Vision. 2009 Aug 18;4(1):1-73.

[5] Yang L, Lu X, Zeng W, Geng W. Trilateral filtering-based retinex for image enhancement. InInternational Conference on Artificial Intelligence and Computational Intelligence 2012 Oct 26 (pp. 400407). Springer, Berlin, Heidelberg.

[6] Tang L, Chen S, Liu W, Li Y. Improved retinex image enhancement algorithm. Procedia Environmental Sciences. 2011 Jan 1;11:208-12.

[7] Jith ON, Babu RV. Joint bilateral filtering based non-local means image denoising. In2014 International Conference on Signal Processing and Communications (SPCOM) 2014 Jul 22 (pp. 1-5) IEEE.

[8] Tang, S., Dong, M., Ma, J., Zhou, Z. and Li, C. Color image enhancement based on retinex theory with guided filter. 29th Chinese Control And Decision Conference (CCDC), 2017, 56765680.

[9] Fu X, Sun Y, LiWang M, Huang Y, Zhang XP, Ding X. A novel retinex based approach for image enhancement with illumination adjustment. In2014 IEEE International Conference on Acoustics, Speech and Signal Processing (ICASSP) 2014 May 4 (pp. 11901194). IEEE.

[10] Le A, Jung SW, Won C. Directional joint bilateral filter for depth images. Sensors. 2014 Jun 26;14(7):11362-78.

[11] Caraffa L, Tarel JP, Charbonnier P. The guided bilateral filter: When the joint/cross bilateral filter becomes robust. IEEE Transactions on Image Processing. 2015 Apr;24(4):1199-208.

[12] Wada N, Kazui M, Haseyama M. Extended joint bilateral filter for the reduction of color bleeding in compressed image and video. ITE Transactions on Media Technology and Applications. 2015;3(1):95-106.

[13] Yang YN, Jiang Z, Yang C, Xia Z, Liu F. Improved retinex image enhancement algorithm based on bilateral filtering. In2015 4th International Conference on Mechatronics, Materials, Chemistry and Computer Engineering 2015 Dec 12. Atlantis Press. 